\title{
Processing resource capacity and the comprehension of garden path sentences
}

\author{
GLORIA S. WATERS \\ McGill University, Montreal, Quebec, Canada \\ and \\ DAVID CAPLAN \\ Massachusetts General Hospital, Boston, Massachusetts
}

\begin{abstract}
Three experiments explored the relationship between verbal working memory capacity and the comprehension of garden path sentences. In Experiment 1, subjects with high, medium, and low working memory spans made acceptability judgments about garden path and control sentences under whole sentence and rapid serial visual presentation (RSVP) conditions. There were no significant differences between subjects with different working memory spans in the comprehension of garden path sentences in either condition. In Experiments $2 \mathrm{~A}$ and 2B, subjects with high and low working memory spans were tested on the same materials at three RSVP rates. There were no significant differences between subjects with different working memory spans in the magnitude of the effect of garden path sentences at any presentation rate. The results suggest that working memory capacity, as measured by the Daneman and Carpenter (1980) reading span task, is not a major determinant of individual differences in the processing of garden path sentences.
\end{abstract}

Research on sentence comprehension has tended to focus on the nature of the representations and computations that are involved in constructing syntactic structures (e.g., Altmann \& Steedman, 1988; Frazier, 1990; Trueswell, Tanenhaus, \& Garnsey, 1994), with little research on the processing resource requirements of this function. However, recently, researchers have become interested in specifying more clearly the nature of the processing resource or working memory system involved in sentence comprehension.

Several pieces of evidence suggest that comprehending sentences requires the allocation of processing resources. Syntactic form affects subjects' accuracy and reaction times in judging the acceptability of sentences (Waters, Caplan, \& Hildebrandt, 1987), and reading times increase at points in a sentence where models of sentence processing predict an increased processing load (King \& Just, 1991). Memory for unrelated material presented during sentence comprehension is affected by syntactic structure (Wanner \& Maratsos, 1978). Aphasic stroke patients' comprehension of sentences is affected by syntactic structure, in a way that suggests a reduction in processing resources available for

This research was supported by grants from the Medical Research Council of Canada (MA9671) and the National Institute of Aging (AG09661). Gloria Waters was supported by a University Research Fellowship from the Natural Sciences and Engineering Research Council of Canada. We wish to thank the action editor, Douglas Hintzman, for extremely detailed and helpful suggestions regarding the organization and style of this paper. D.C. is affiliated with the Neuropsychology Laboratory at Massachusetts General. Correspondence concerning this article should be addressed to G. S. Waters, School of Communication Sciences and Disorders, McGill University, 1266 Pine Ave. W., Montreal, PQ, Canada H3G 1A8. syntactic processing (Caplan, Baker, \& Dehaut, 1985; Caplan \& Hildebrandt, 1988).

How specialized is the processing resource system required for sentence comprehension? Many linguists and psycholinguists have argued that the processes involved in sentence comprehension are distinct from those involved in other verbally mediated functions, such as reasoning and problem solving (e.g., Fodor, 1983). Claims regarding the "modularity" of sentence processing have generally been restricted to information-processing aspects of this function. However, a parallel question arises about the processing resources involved in sentence comprehension: are they specific to sentence comprehension (or to aspects of the sentence comprehension process) or are the same processing resources used in sentence comprehension and other verbal tasks?

Just and Carpenter (1992) have approached the question of the nature of the processing resources used in sentence comprehension by attempting to determine whether individual differences in performance on a task that was designed to measure verbal working memory capacity - the Daneman and Carpenter (1980) reading span task-are related to individual differences in the ability to structure sentences syntactically. They have interpreted the results of a variety of experiments as showing that individuals with lower reading spans are less efficient at structuring sentences syntactically than are subjects with higher spans. On this basis, they have argued that the working memory system that is measured by the reading span task is involved in syntactic processing during sentence comprehension. We have questioned this "capacity theory" on several grounds. At an empirical level, many results that form the basis for Just and Carpenter's claims are not statistically 
significant, and, at a theoretical level, their model does not generate clear predictions (Waters \& Caplan, in press).

Just and Carpenter's (1992) capacity theory is also contradicted by studies of two different types of brain-damaged patients. Patients with reduced working memory (e.g., patients with Alzheimer's disease) are not more impaired than normals in comprehending syntactically more complex sentences (Rochon, Waters, \& Caplan, 1994), even under dual-task conditions that load working memory (Waters, Caplan, \& Rochon, 1995). Conversely, patients with aphasia due to stroke, who suffer from a reduction in the processing resources that are needed for syntactic processing, are not more affected by a digit load than normals when comprehending syntactically more complex sentences (Caplan \& Waters, 1994). Both these results suggest that the resource system used in syntactic processing in sentence comprehension is separate from that used in the Daneman and Carpenter (1980) reading span task.

Given the results of the studies of brain damaged patients, the differences in sentence processing that Just, Carpenter and their colleagues claim to have found in normal subjects with different reading spans provide crucial empirical support for the capacity theory. In this paper, we focus on one aspect of this support: subjects' abilities to comprehend sentences with syntactic garden paths. Garden path sentences are locally ambiguous syntactic structures, whose ultimate resolution is toward an unfavored syntactic representation. Readers and listeners are frequently misled as to the syntactic structure of garden path constructions. Perhaps the best known garden path structure is illustrated in (1) (Bever, 1970):

\section{The boat floated down the river sank.}

Readers (and listeners) generally assign the boat as the subject of floated in (1), and have considerable difficulty revising this assignment in favor of one in which the boat is the subject of sank, and floated down the river is a reduced relative clause.

Garden path sentences are of interest with respect to the role of processing resources, since they are intuitively difficult and would be expected to require more processing resources to be interpreted correctly than would unambiguous sentences with the same structure. Therefore, if individual differences in the availability of processing resources affect sentence comprehension, there should be differences in the ability of subjects with high and low processing capacity to interpret these sentences.

MacDonald, Just, and Carpenter (1992) have explored the processing of the type of garden path sentences illustrated in (1) by readers who have high and low working memory capacities as measured by the Daneman and Carpenter (1980) reading span task. MacDonald et al. used a self-paced word-by-word reading task, in which each sentence was followed by a question. They presented garden path sentences such as ( $2 a)$ and non-garden path control sentences such as $(2 \mathrm{~b})$.

2a. The experienced soldiers warned about the dangers conducted the midnight raid 2b. The experienced soldiers who were told about the dangers conducted the midnight raid.

They found that subjects took longer to read the final words and made more errors in answering questions about garden path sentences (2a) than in answering questions about non-garden path sentences, confirming the difficulty of these garden path sentences. Low-capacity subjects made more errors in answering questions about garden path than about non-garden path sentences, while high-capacity subjects took longer to read the words in the final phrases of garden path sentences than in those of non-garden path sentences. MacDonald et al. interpreted these results as consistent with the capacity theory of sentence comprehension. They argued that both low- and highspan readers attempt to construct both the preferred and the unpreferred syntactic structures in sentences like (2a), but that low-span subjects cannot maintain both structures in working memory. Therefore, low-capacity subjects make more errors on garden path sentences, because they do not have the unpreferred syntactic structure available at the end of the sentence, whereas high-span subjects take longer to read the words in the final phrases of garden path sentences, because they maintain both the preferred and the unpreferred syntactic structures throughout the sentence.

However, there are several problems with this interpretation of MacDonald et al.'s (1992) results. First, the effects described above were not reliable statistically over subjects. Second, there were only six sentences in each condition, and subjects made errors on $35 \%-50 \%$ of the garden path sentences, so that the reaction time analysis for garden path sentences was based on data for approximately three sentences. Third, presumably because of the small number of sentences of each type, item statistics were not reported. Finally, the pattern of results could simply have reflected different speed-accuracy tradeoffs in the high- and low-span groups.

Because of the ambiguity of the MacDonald et al. (1992) results, we undertook further study of the relationship between working memory and comprehension of garden path sentences. In Experiment 1 we tested the ability of 65 subjects to process garden path sentences. The stimuli in this experiment consisted of sentences with reduced relative clauses, as had been tested in the MacDonald et al. study, and two additional syntactic structures-sentential complements and embedded clauses--which have been shown to result in garden path effects. Subjects made sentence acceptability judgments about acceptable and unacceptable garden path sentences and two types of nongarden path sentences under two different presentation conditions. In the whole sentence condition, the sentence remained on the screen until the subject had responded. In the RSVP (rapid serial visual presentation) condition, the words of the sentence were presented sequentially in the center of a computer screen. Miyake, Carpenter, and Just (1994) have argued that the severe time constraints imposed by this technique reduce the processing resources available for syntactic processing. Moreover, since the stimulus is not present for the subject to review in the RSVP 
condition, this condition is expected to have a greater memory load than the whole sentence condition. If Just and Carpenter's (1992) theory is correct, low-span subjects should be more impaired than high-span subjects in judging garden path sentences, and this difference should be larger under RSVP than in the whole-sentence conditions.

\section{EXPERIMENT 1}

\section{Method}

\section{Subjects}

Sixty-five subjects participated in the study $(25$ males and $40 \mathrm{fe}$ males). They ranged from 50 to 80 years of age, with a mean of 64.5 years. All subjects had at least a high school education, and the majority had an undergraduate university education. All subjects were healthy individuals, were considered to be aging normally, had normal or corrected-to-normal vision, and spoke English as their mother tongue.

\section{Materials}

Reading span task. The materials for the reading span task were a subset of those used in Waters et al. (1987). They consisted of 50 semantically acceptable and 50 semantically unacceptable sentences. The sentences were all of the same syntactic structure (cleft subject). Half had verbs that required animate subjects (c.g., It was the man that clenched the pillow) and half had verbs that required animate objects (e.g., It was the toy that fascinated the child). Unacceptable sentences were formed by inverting the animacy of the subject and object noun phrase (e.g., It was the pillow that clenched the man). The sentences were all $8-11$ words in length $(M=8.7$ for acceptable, 8.1 for unacceptable). The 100 sentences were divided into five sets corresponding to the Span Sizes 2-6, with five trials at each span size. Thus, the stimuli for Span Size 2 consisted of 10 sentences, Span Size 3 of 15 sentences, etc.

Sentence acceptability task. The stimuli for the sentence acceptability task consisted of 450 sentences as outlined in Table 1 . Of these, 270 sentences were acceptable and 180 were unacceptable. One third of the sentences of each type included sentential complements, one third included reduced relative clauses, and one third included embedded clauses. For the acceptable sentences, for each structure there were 30 garden path sentences and 60 non-garden path sentences. The length of the garden path segment in each of these structures was four to five words and thus corresponded to the length that MacDonald et al. (1992) claim results in the greatest differences between memory span groups.

One half of the non-garden path sentences included an overt marker of the target structure (non-garden path A), and the other half included lexical items whose morphological forms were such that they that did not result in a garden path structure (non-garden path B). These two types of control sentences were included to ensure that the garden path sentences were more demanding than sentences in which the critical structures were marked by either a function word or morphological cues.

It was not possible to match the sentences across the three structures for length because of inherent differences between them. How ever, within a type of structure, the garden path and non-garden path sentences were matched as closely as possible. The mean number of words for the garden path, non-garden path $A$ and non-garden path $B$ sentences were $13.5,13.8$, and 13.5 for sentential complements; $7.1,8.0$, and 7.1 , for the reduced relatives; and $8.3,8.8$, and 8.3 for the embedded clauses. Non-garden path A sentences were longer because of the additional function word used to unambiguously indicate the syntactic structure.

The unacceptable sentences consisted of 30 garden path and 30 non-garden path sentences of each structure. These sentences were unacceptable due to an anomalous word. The location of the anomalous word was varied across sentences.
Table 1

Examples of the Sentences Used in the Experiment

\begin{tabular}{|c|c|}
\hline Sentence Type & Sentence \\
\hline \multicolumn{2}{|c|}{ 1. Sentential Complement } \\
\hline \multicolumn{2}{|l|}{ Acceptable } \\
\hline $\begin{array}{l}\text { Garden path } \\
(n=30)\end{array}$ & $\begin{array}{l}\text { The defendant confided to the lawyer he ad- } \\
\text { mired the judge was his brother. }\end{array}$ \\
\hline $\begin{array}{l}\text { Non-garden path } \mathrm{A} \\
(n=30)\end{array}$ & $\begin{array}{l}\text { Anna wrote to the congressman the voters } \\
\text { supported that his policies were unfair. }\end{array}$ \\
\hline $\begin{array}{l}\text { Non-garden path B } \\
(n=30)\end{array}$ & $\begin{array}{l}\text { The farmer agreed with the guest his wife } \\
\text { had invited the field should be ploughed. }\end{array}$ \\
\hline \multicolumn{2}{|l|}{ Unacceptable } \\
\hline $\begin{array}{l}\text { Garden path } \\
(n=30)\end{array}$ & $\begin{array}{l}\text { The chef declared to the reporters he baked } \\
\text { the public would be impressed. }\end{array}$ \\
\hline $\begin{array}{l}\text { Non-garden path } \\
(n=30)\end{array}$ & $\begin{array}{l}\text { The boss read the memo the secretary typed } \\
\text { that the reports were fired. }\end{array}$ \\
\hline
\end{tabular}

2. Reduced Relative

Acceptable

Garden path

$(n=30)$

Non-garden path A The farmer who was leased a tractor

$(n=30)$

Non-garden path B The boy given a cookie was hungry.

$(n=30)$

Unacceptable

Garden path

$(n=30)$

Non-garden path

$(n=30)$

The horse raced past the barn fell.

3. Embedded Clause

Acceptable

Garden path

$(n=30)$

Non-garden path $\mathrm{A}$

$(n=30)$

Non-garden path $\mathrm{B}$

$(n=30)$

Unacceptable

Garden path

$(n=30)$

Non-garden path

$(n=30)$

The cookie fried the oven ate.

The waiter who was tipped a wig quit.

The picture books were lying beside was a landscape.

The ocean that the fish swam in was polluted.

The house Bill was sitting in belonged to foreigners.

The commercial photographers shot on vacation modelled.

The football that the champions tossed around prayed.

The stimuli were randomized in four blocks, with an equal number of sentences of each type in each block.

\section{Procedure}

Subjects were tested over 2 sessions on two separate days. All subjects were first tested on the reading span task in order to obtain a measure of their working memory capacity. They were then randomly assigned to and tested on one condition (whole sentence or RSVP) of the sentence acceptability judgment task. They were tested on the remaining condition in the following session.

Reading span task. Subjects were tested on a block of practice trials and then on five test trials at each of the Set (Span) Sizes 2-6. On each trial, an asterisk appeared on the video screen, followed 200 msec later by the first sentence in the set. The subject read the sentence silently and pushed the right response key if the sentence was acceptable and the left response key if it was unacceptable. As soon as a decision about one sentence had been made, the next sentence in the set appeared. When the subject had made a decision about all of the sentences in the set, an asterisk appeared to indicate to the subject that the last word of all the sentences in the set was to be re- 
called. Subjects were instructed to perform the sentence task very accurately and then to perform as well as they could on the recall task. ${ }^{1}$ They were instructed to recall the words in the correct serial order, and credit was given only for trials in which items were recalled in the correct serial order. A subjects' reading span was defined as the largest set size at which all of the words in the correct serial order on three of the five trials were correctly recalled. An additional 0.5 was added to the subject's score if two of the five words in the next highest set were recalled (King \& Just, 1991). Subjects were not tested at Span Size 1. Subjects who recalled fewer than 2 trials correctly at Span Size 2 were considered to have a span of 1. Those who recalled 2 trials correctly at Span Size 2 were assigned working memory spans of 1.5 . In addition, subjects' accuracy on the sentence acceptability portion of the working memory span task was scored in terms of the percent of judgments that were correct on the sentences presented at their span. ${ }^{2}$

Sentence acceptability task. Subjects were tested on the sentence acceptability task under two presentation conditions, whole sentence and rapid serial visual presentation (RSVP), on different days, with the order of presentation of the conditions randomized across subjects. Subjects were given the following instructions in both the whole sentence and RSVP conditions:

In this experiment, we want you to read each sentence as it appears on the screen. Read it carefully, and decide if it is a "good" sentence in English. That is, does it make sense. If it does make sense, press the right key marked $\mathrm{Y}$ for yes. Similarly, if it does not make sense, press the left key marked $\mathrm{N}$ for no.

We will start with a small practice session.

In the whole-sentence condition, each sentence appeared individually in mixed case on the screen of an IBM PS2/50 computer and remained on the screen until the subject indicated whether it was acceptable by pressing one of two keys on the keyboard. Stimulus presentation was controlled by the Micro Experimental Laboratory system and the computer recorded the time from the presentation of the sentence until the subject responded. In the RSVP condition, the words of the sentence were displayed successively for $250 \mathrm{msec}$ in the center of the computer screen. The screen became blank after the last word. Subjects responded by pressing one of two response keys on the keyboard, and response times were recorded from the offset of the final word of the sentence. The next sentence was displayed $1 \mathrm{sec}$ after the response.

\section{Results}

\section{Reading Span Data}

Subjects were divided into three groups - high, medium and low working memory span-on the basis of their performance on the reading span task using the King and Just (1991) criteria. Table 2 shows the characteristics of the three span groups. One-way analyses of variance (ANOVAs) showed that the three groups did not differ in terms of age $[F(2,62)=1.7$, n.s. $]$. As might be expected, however, there were small differences across the groups in years of education $[F(2,62)=3.9, p<.05]$ and WAIS vocabulary scores $[F(2,60)=3.2, p<.05]$. On both these measures, the lowspan subjects differed significantly from the high-span subjects, but the medium subjects did not differ significantly from either the high- or the low-span subjects. In addition, all three subject groups differed significantly in reading spans as measured by the final word recall measure, but did not differ in accuracy on the sentence acceptability component of the reading span task. ${ }^{3}$
Table 2

Characteristics of Subjects in the Memory Span Groups: Experiment 1

\begin{tabular}{|c|c|c|c|}
\hline & \multicolumn{3}{|c|}{ Memory Span Group } \\
\hline & $\begin{array}{c}\text { Low } \\
(n=18)\end{array}$ & $\begin{array}{l}\text { Medium } \\
(n=21)\end{array}$ & $\begin{array}{c}\text { High } \\
(n=26)\end{array}$ \\
\hline \multicolumn{4}{|c|}{ Age (Yrs) } \\
\hline$M$ & 66.3 & 65.1 & 62.0 \\
\hline$S D$ & 7.9 & 8.3 & 8.1 \\
\hline Range & $51-80$ & $51-78$ & $50-78$ \\
\hline \multicolumn{4}{|c|}{ Education (Yrs) } \\
\hline$M$ & 16.4 & 16.7 & 17.7 \\
\hline$S D$ & 1.9 & 1.3 & 1.7 \\
\hline Range & $12-20$ & $14-19$ & $15-21$ \\
\hline \multicolumn{4}{|c|}{ WAIS Vocabulary (Raw Score) } \\
\hline$M$ & 61.6 & 62.8 & 65.2 \\
\hline$S D$ & 4.7 & 6.2 & 3.2 \\
\hline Range & $53-69$ & $47-70$ & $56-70$ \\
\hline \multicolumn{4}{|c|}{ Reading Span (Final Word Recall) } \\
\hline$M$ & 1.8 & 3.1 & 4.6 \\
\hline$S D$ & .5 & .3 & .6 \\
\hline Range & $1.0-2.5$ & $3.0-3.5$ & $4.0-6.0$ \\
\hline \multicolumn{4}{|c|}{ ( $\%$ Correct on Acceptability Judgment) } \\
\hline$M$ & 95.2 & 95.4 & 95.8 \\
\hline$S D$ & 4.6 & 5.6 & 6.5 \\
\hline Range & $84-100$ & $84-100$ & $84-100$ \\
\hline
\end{tabular}

\section{Sentence Acceptability Task}

Discrimination of acceptable and unacceptable sentences. Subjects' overall levels of performance were assessed by using $A^{\prime}$ measures. $A^{\prime}$ scores were calculated separately for each subject for each condition (whole sentence vs. RSVP), structure (complement, reduced relative, embedded), and type of sentence (garden path, nongarden path $\mathrm{A}$, non-garden path $\mathrm{B}$ ). $A^{\prime}$ is a nonparametric index of sensitivity that can be used to isolate the criterion (or bias) factor from the measure of sensitivity to the acceptability of a sentence (Pollack \& Norman, 1964). The $A^{\prime}$ statistic is based on the estimated area under the receiver-operating characteristic (ROC) curve and is theoretically equal to the proportion of correct responses that can be obtained in a two-alternative forced choice task. It is thus quite easily interpreted; for example, an $A^{\prime}$ value of .95 would be equivalent to an expected score of $95 \%$ on a two-alternative forced choice task.

The $A^{\prime}$ scores were analyzed in a 3 (memory span: low, medium, high) $\times 2$ (condition: whole sentence, RSVP) $\times$ 3 (structure: complement, relative, embedded) $\times 3$ (garden path status: garden path, non-garden path A, nongarden path B) ANOVA with memory span as a betweensubjects factor and the other factors as within-subjects factors. Because of the complexity of the design, in this and subsequent analyses reported in this paper, the per comparison alpha level was set at $p<.01$ in order to reduce the probability of a Type I error. Tukey post hoc tests were carried out to analyze the source of significant main effects and interactions. 

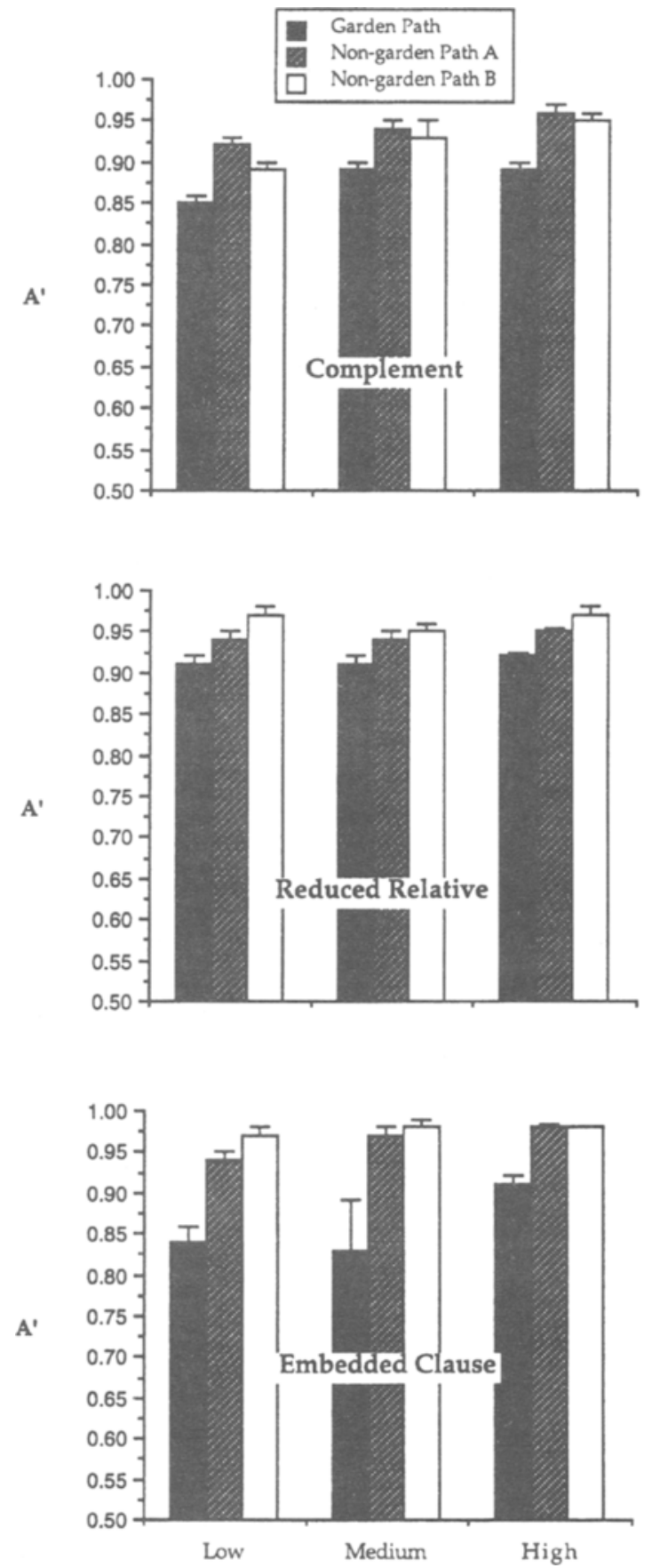

Figure 1. Mean $A^{\prime}$ scores (and standard deviations) for each memory span group (low, medium, high) and sentence type in the whole-sentence presentation condition of Experiment 1.

Figure 1 shows the data from the whole sentence condition and Figure 2 shows the data from the RSVP condition. The ANOVA revealed a significant main effect of memory span $[F(2,62)=9.4, p<.001]$. Low-span subjects (mean $A^{\prime}=.89$ ) were less sensitive to the acceptability of sentences than were medium- (.92) or high-span subjects (.93), who did not differ from one another. In addition, there were significant main effects of condition $[F(1,62)=$ $29.6, p<.001]$, structure $[F(2,124)=50.1, p<.001]$, and garden path status $[F(2,124)=248.7, p<.001]$. These effects were due to poorer performance in the RSVP (mean $\left.A^{\prime}=.90\right)$ than in the whole sentence condition $(.93)$, on complement structures $(.89)$ than on sentences with either relative (.93) or embedded structures (.92), and on garden path sentences (.87) than on non-garden path sentences (non-garden path $\mathrm{A}=.93$, non-garden path $\mathrm{B}=.94$ ).

These main effects were qualified by significant interactions between memory span and structure $[F(4,124)=$
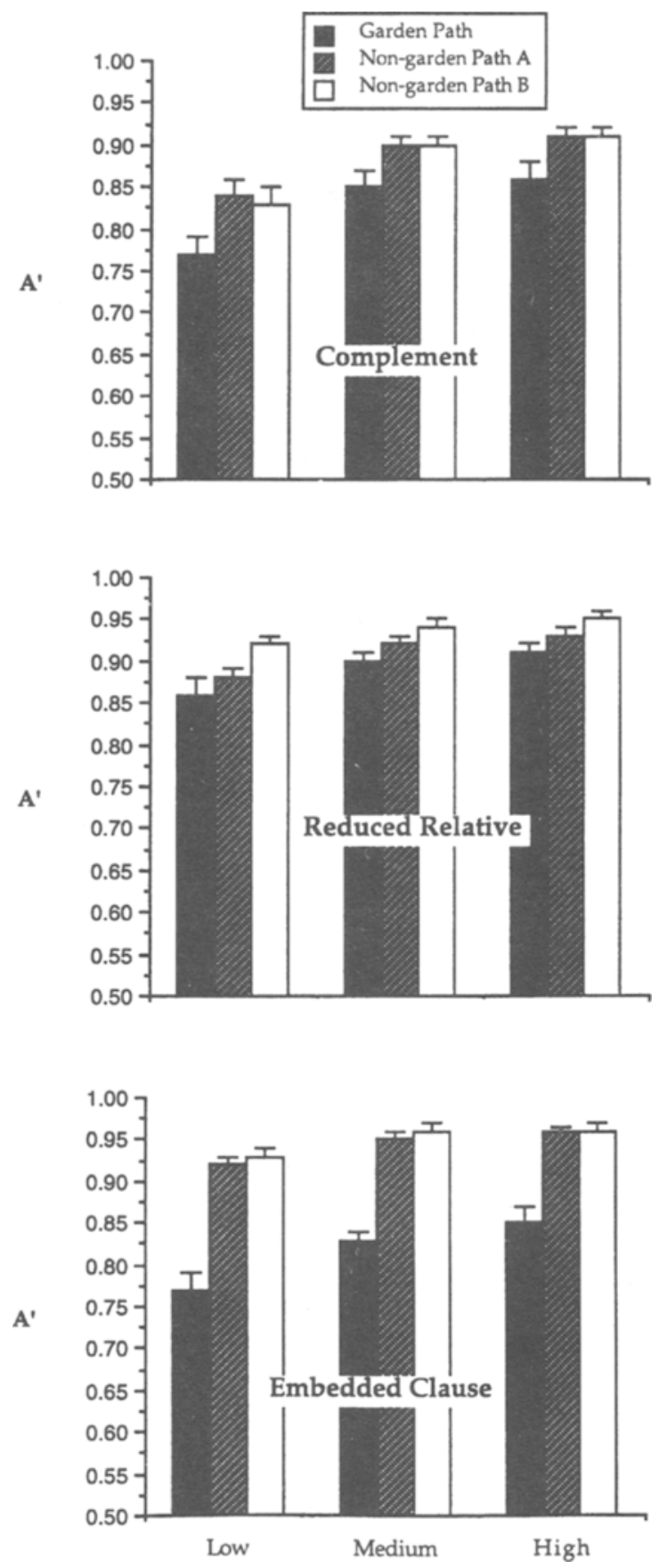

Figure 2. Mean $A^{\prime}$ scores (and standard deviations) for each memory span group (low, medium, high) and sentence type in the RSVP presentation condition of Experiment 1. 
$4.7, p<.01]$, structure and condition $[F(2,124)=6.4, p<$ $.01]$, and structure and garden path status $[F(4,248)=33.3$, $p<.001]$. Analysis of the interaction between memory span and structure showed that the pattern of poorer performance on complements than on the other two sentence types was statistically reliable only for the low-span subjects. For the medium- and high-span subjects, performance was comparable across the three structures. Analysis of the condition $X$ structure interaction showed that the pattern of performance across the three structures outlined above (complement $<$ relative $=$ embedded) held in both the whole-sentence and RSVP conditions. Analysis of the interaction showed that all possible pairwise comparisons of performance across the whole sentence and RSVP conditions were significant other than that between performance on complement sentences in the wholesentence condition and embedded sentences in the RSVP condition. Finally, analysis of the interaction between garden path status and structure showed that the pattern of performance outlined above (garden path < non-garden path $A=$ non-garden path $B$ ) held only for the complement and embedded structures. For the relative structures, performance was poorer on garden path sentences than on non-garden path A sentences, which in turn was poorer than performance on non-garden path $B$ sentences. Neither the two-way interactions between memory span and condition, or memory span and garden path status, or any of the three-way interactions involving the memory span, condition, structure, and garden path status factors, or the four-way interaction approached significance (all $p \mathrm{~s}>.1$ ).

Reaction times for acceptable sentences. Since the data of principal interest concerned performance on the acceptable sentences, the data for the acceptable sentences were analyzed further. For these sentences, the mean percent correct and mean reaction time for correct responses were calculated separately for each subject group for each condition, syntactic structure, and sentence type. For each subject, extreme reaction time values within each condition (those less than or greater than the condition mean \pm three standard deviations) were replaced by the mean of the condition. The analyses of the percent correct scores for acceptable sentences were virtually identical to the results from the $A^{\prime}$ analysis in all experiments reported in this paper and have not been presented here because of space limitations.

As noted above, reaction times for the whole-sentence condition included the reading time for the sentence, whereas those for the RSVP condition included the reading time of only the last word of the sentence. Thus, the reaction time data for the whole-sentence and RSVP conditions were analyzed separately. Since the three different sentence structures differed in length, the total reading time for each sentence in the whole-sentence condition was divided by the number of words in the sentence to allow the three structures to be compared. The data from each condition were analyzed in separate 3 (memory span) $\times$ 3 (structure) $\times 3$ (garden path status) ANOVAs with both subject and item means as units. Only the effects that were significant in both the subject and item analyses are presented here.

Figure 3 shows the mean reading times per word for correct responses in the whole sentence condition. The ANOVAs showed that there was a significant main effect of garden path status $\left[F_{1}(2,124)=270.3, p<.001\right.$; $\left.F_{2}(2,261)=101.1, p<.001\right]$ and a significant structure $\times$ garden path status interaction $\left[F_{1}(4,248)=64.8, p<.001\right.$; $\left.F_{2}(4,261)=9.2, p<.001\right]$. Reading times were longer for garden path sentences than for non-garden path $A$ or $B$ sentences. The interaction was due to the fact that performance on non-garden path A and non-garden path B sentences did not differ for complements and relatives, but reading times were longer for non garden path $A$ than for non-garden path B sentences with embedded clauses.

The data for the RSVP condition are presented in Fig. ure 4. The ANOVAs showed a significant main effect of garden path status $\left[F_{1}(2,124)=136.7, p<.001 ; F_{2}(2,261)=\right.$ 99.6, $p<.001]$ and an interaction between structure and garden path status $\left[F_{1}(4,248)=34.9, p<.001 ; F_{2}(4,261)=\right.$ $11.5, p<.001]$. Reaction times were longer for garden path sentences than for both types of non-garden path sentences, and reaction times were longer to non-garden path A sentences than to non-garden path B sentences. Analysis of the interaction showed that this pattern held for sentences with reduced relatives, but performance on the two types of non-garden path sentences did not differ for sentences with complements and embedded clauses.

There were no effects of group in either condition.

Correlations between garden path effects and memory span. In a further effort to determine whether there was a relationship between memory span and the extent to which subjects were affected by garden path structures, correlations were carried out between memory span and the magnitude of the garden path effect seen in each condition. Mean proportional increases in reaction time on correct garden path compared with correct non-garden path structures were calculated for each subject for each condition (i.e., [garden path - non-garden path A]/nongarden path $A$; [garden path - non-garden path $B$ ]/nongarden path B). The correlations between memory span and the magnitude of the garden path effect seen in both the whole-sentence $(r=.12)$ and the RSVP $(r=.20)$ conditions were not significant. Differences in errors on garden path compared with non-garden path sentences were also calculated for each condition. The correlation between memory span and these difference scores failed to reach significance in both the whole-sentence $(r=.17)$ and RSVP $(r=.03)$ conditions.

\section{Discussion}

The results confirm other reports that it is harder to process garden path sentences than syntactically similar control sentences. Garden path effects were found for all three sentence types tested in this experiment. They arose in comparing garden path sentences against two different types of control sentences, and in both the whole-sentence and RSVP presentation conditions. Also as expected, the RSVP 

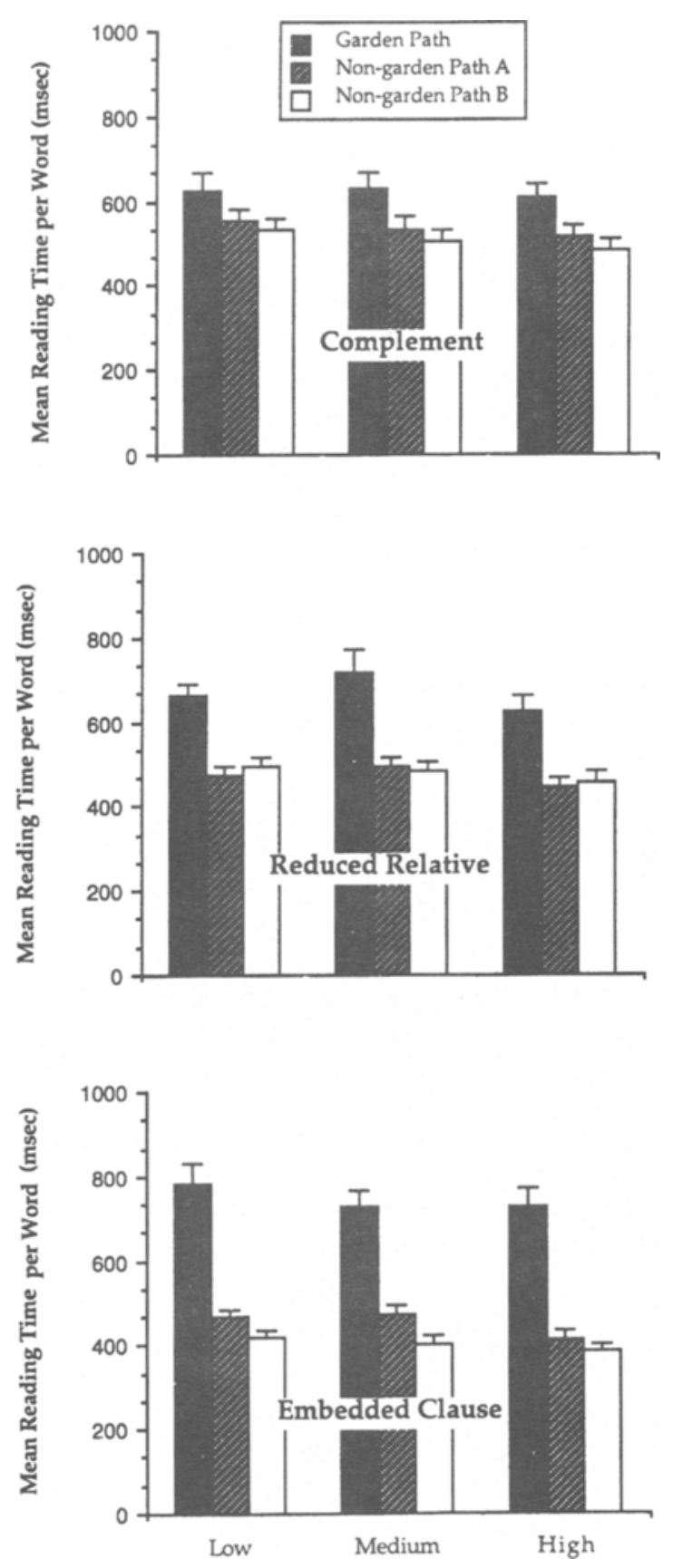

Figure 3. Mean reading times per word (and standard deviations) for correct responses for each memory span group (low, medium, high) and sentence type in the whole-sentence presentation condition of Experiment 1.

condition was more difficult than the whole-sentence condition. The increased difficulty of the RSVP condition was likely due to the increased memory load in the RSVP condition, as well as the novelty of the RSVP task.

Despite the difficulty in making judgments about garden path sentences, especially in the more demanding RSVP condition, both direct comparisons of the performance of subjects differing in working memory capacity and corre- lational analyses indicate that working memory capacity did not determine performance in processing garden path sentences. The overall $A^{\prime}$ of low-span subjects was lower than that of high-span subjects, indicating that the lowspan subjects were not as efficient as the high-span subjects at making acceptability judgments. However, the lowspan subjects were not worse than the high-span subjects
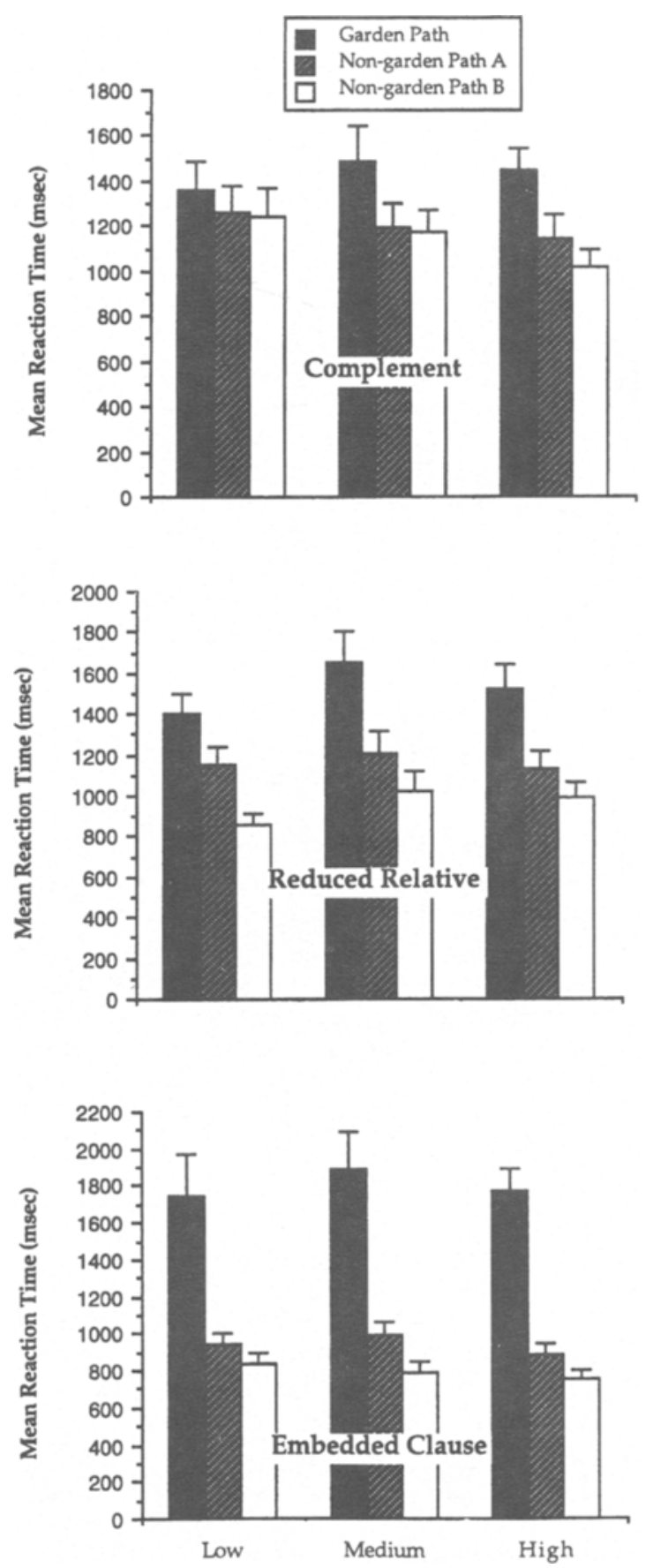

Figure 4. Mean reaction times (and standard deviations) for correct responses for each memory span group (low, medium, high) and sentence type in the rapid serial visual presentation condition of Experiment 1. 
in processing garden path sentences in either the wholesentence or the RSVP condition, indicating that their increased difficulty with the task is unlikely to be due to difficulties with syntactic processing.

There was some indication that the different span groups were differentially affected by the sentence type variable. In the $A^{\prime}$ analysis, only the low span subjects performed more poorly on the complement structures. Since complement sentences were more difficult than embedded or relative clause sentences, this pattern might suggest that lowspan subjects were more affected than the other groups by syntactic complexity. However, the complement sentences were also longer than the other two sentence types, making it impossible to determine whether the poorer performance of the low-span subjects on this sentence type was due to length or syntactic structure. We note that the principal means of increasing syntactic processing in this experiment was the garden path variable, not the sentence type variable. If syntactic structure affected groups differently, its effects would be expected to be seen in the comparison of garden path and non-garden path sentences, which was not the case. Thus, the group $\times$ sentence type interaction found in the $A^{\prime}$ data is not likely to have been due to inefficient syntactic processing in the low-span subjects.

The results of this experiment suggest that low-span subjects are not less efficient at syntactic processing than high-or medium-span subjects. However, it is possible that differences between high- and low-span subjects might emerge if the task were more demanding. In order to further investigate this possibility, we carried out two additional experiments in which we manipulated the rate at which the words were presented in the RSVP condition.

\section{EXPERIMENTS 2A AND 2B}

In Experiments $2 \mathrm{~A}$ and 2B, subjects were tested under RSVP conditions using the same stimulus materials as in Experiment 1. In Experiment 2A, subjects were tested at the same rate as in Experiment 1 ( $250 \mathrm{msec}$ per word) and at a faster rate ( $170 \mathrm{msec}$ per word). In Experiment 2B, subjects were tested at the rate of $120 \mathrm{msec}$ per word.

Two additional changes were made in Experiments 2A and $2 \mathrm{~B}$ that were designed to make the studies more comparable to the MacDonald et al. (1992) study. First, the subjects in these experiments were college students as in MacDonald et al., rather than elderly individuals as in Experiment 1 . Second, subjects were classified as high versus low span with the use of the original Daneman and Carpenter (1980) reading span task and stimulus materials, rather than the task and stimulus materials from Waters et al. (1987).

\section{Method}

\section{Subjects}

Fifty subjects ( 16 males and 34 females) were chosen from a larger pool of subjects on the basis of their scores on the Daneman and Carpenter (1980) reading span tasks for Experiment 2A, and 32 subjects ( 7 males and 25 females) were chosen for Experiment $2 \mathrm{~B}$. Half of the subjects in each experiment had low working memory spans and half had high spans, according to the same criteria as those in the MacDonald et al. study (see below). None of the subjects had participated in Experiment 1. Subjects ranged in age from 18 to 34 years. The mean ages of the low-and high-span subjects were 24.4 and 23.9 years in Experiment 2A, and 20.5 and 21.6 in Experiment $2 \mathrm{~B}$. All subjects had at least a high school education, and the majority of the subjects were attending McGill University. The average years of education for the low- and high-span subjects were 15.9 and 16.5 in Experiment 2A, and 15.3 and 14.6 in Experiment 2B. Results of $t$ tests showed that there were no significant differences in the high- and low-span subjects in age or education in either experiment. All subjects had normal or corrected-to-normal vision.

\section{Procedures and Materials}

All subjects were tested on the reading span task in a preliminary session. For Experiment 2A, subjects who fell into either the highor the low-span group were tested in two additional sessions on 2 separate days. Half of the subjects in each span group were tested in the $170-\mathrm{msec}$ condition first and the $250-\mathrm{msec}$ condition second, and the other half were tested in the reverse order. For Experiment 2B, subjects were tested on the sentence acceptability task with the rate of presentation being $120 \mathrm{msec}$ per word in one additional session.

Reading span task. Subjects' reading spans were tested with the methods and materials from Daneman and Carpenter (1980, Experiment 1). This task is very similar to the task used in Experiment 1 , other than the fact that subjects are required to read a series of sentences aloud at their own pace rather than make judgments about a series of sentences, before recalling the last word of each sentence in the series. The instructions to the subjects were identical to those used by Daneman and Carpenter. The subjects were told that they should write down the words in the order in which they saw them, but that if they could not remember all of the words or the order in which they were presented, they should recall whatever words they could remember. They also were instructed not to begin with the last word presented.

The stimuli were 100 unrelated sentences, ranging in length from 13 to 16 words. Each sentence was typed on a single line of an index card and the cards were arranged in five sets each of two, three, four, five, and six sentences. Each sentence in the series ended in a different word. The beginning and end of each set were marked by blank cards.

Reading span was calculated as in Experiment 1. As in MacDonald et al. (1992), subjects with reading spans of 3.5 or greater were considered to be high-span subjects; those with spans of 2.5 or less, to be low-span subjects.

Sentence acceptability judgment task. The stimuli for the sentence acceptability judgment task were the same as those used in Experiment 1 . For Experiment $2 \mathrm{~A}$, the total pool of stimulus sentences was divided in half, with an equal number of sentences of each type (acceptable and unacceptable; garden path, non-garden path A, nongarden path B; complement, reduced relative, embedded) in each half. One set of stimulus sentences was used in the 170 -msec presentation condition and the other in the $250-\mathrm{msec}$ condition. The stimuli in each set were randomized in four blocks, with an equal number of sentences of each type in each block. In Experiment 2B, all subjects were tested on the full set of stimulus materials from Experiment 1 . The instructions to the subjects in both experiments were identical to those in Experiment 1.

\section{Results}

\section{Experiment 2A}

Discrimination of acceptable and unacceptable sentences. Figure 5 presents the $A^{\prime}$ scores for the two groups of subjects at the two presentation rates. These data were analyzed in a 2 (memory span) $\times 2$ (presentation rate) $\times 3$ (structure) $\times 3$ (garden path status) ANOVA. The $A^{\prime}$ scores of low-span subjects (mean $A^{\prime}=.81$ ) were signif- 
icantly lower than those of high-span subjects (.86) $[F(1,48)=6.8, p<.01]$. Subjects were less sensitive to the acceptability of garden path sentences (mean $A^{\prime}=.78$ ) than to either type of non-garden path sentence (for nongarden path A sentences, .86; for non-garden path B sentences, .87) $[F(2,96)=61.6, p<.001]$. Subjects were less sensitive to the acceptability of sentences with complement structures (mean $A^{\prime}=.79$ ) than to sentences with either reduced relative $(.86)$ or embedded clause $(.85)$ structures $[F(2,960=35.8, p<.001]$.

There were significant two-way interactions between presentation rate and structure $[F(2,96)=8.7, p<.001]$, presentation rate and garden path status $[F(2,96)=7.9, p<$ $.001]$, and structure and garden path status $[F(4,192)=$ $15.6, p<.001]$. Analysis of these interactions showed that the effects of garden path status and structure outlined above held at both presentation rates. The presentation rate $\times$ structure interaction resulted from performance on the complement structure, but not the other sentences, being lower in the 170 - than in the 250 -msec presentation condition. The presentation rate $\times$ garden path interaction resulted from lower $A^{\prime}$ s in garden path sentences at the 250 -msec than at the $170-\mathrm{msec}$ rate, and lower $A^{\prime} \mathrm{s}$ in nongarden-path A sentences at the 170 -msec than at the 250 msec rate. The structure $\times$ garden path status interaction arose because garden path sentences differed from nongarden path B sentences for all three structures, but only differed from non-garden path A sentences for the complement and embedded structures. The two-way interaction between structure and garden path status was further qualified by a three-way interaction between presentation rate, structure, and garden path status $[F(4,192)=3.8, p<$ $.01]$. Post hoc analysis of this interaction showed that, in the 250-msec presentation condition, there were garden path effects for sentences with complement and embedded structures, but that in the 170 -msec presentation condition, garden path effects were seen only for sentences with embedded structures.

Reaction times for acceptable sentences. The mean reaction times for correct responses are shown in Figure 6. ANOVAs followed by post hoc tests showed that reaction times were significantly longer for complements than for either embedded or reduced relative sentences $\left[F_{1}(2,90)=\right.$ $\left.12.6, p<.001 ; F_{2}(2,252)=15.8, p<.001\right]$. These latter two sentence types did not differ from one another. However, this effect of structure was qualified by a two-way interaction between presentation rate and structure $\left[F_{1}(2,90)=\right.$ $\left.9.7, p<.001 ; F_{2}(2,252)=4.5, p<.01\right]$. Analysis of this interaction showed that the effect of structure occured only at the 250 -msec presentation rate. There were no significant differences across the three structures at the faster (170$\mathrm{msec}$ ) presentation rate.

Reaction times were also longer for garden path than for both types of non garden path sentences $\left[F_{1}(2,90)=57.6\right.$, $\left.p<.001 ; F_{2}(2,252)=86.7, p<.001\right]$. Although this effect was qualified by an interaction between structure and garden path status $\left[F_{1}(4,180)=24.4, p<.001 ; F_{2}(4,252)=6.7, p<\right.$ $.001]$, post hoc analysis of the interaction showed that there were garden path effects for all three structures. The inter-
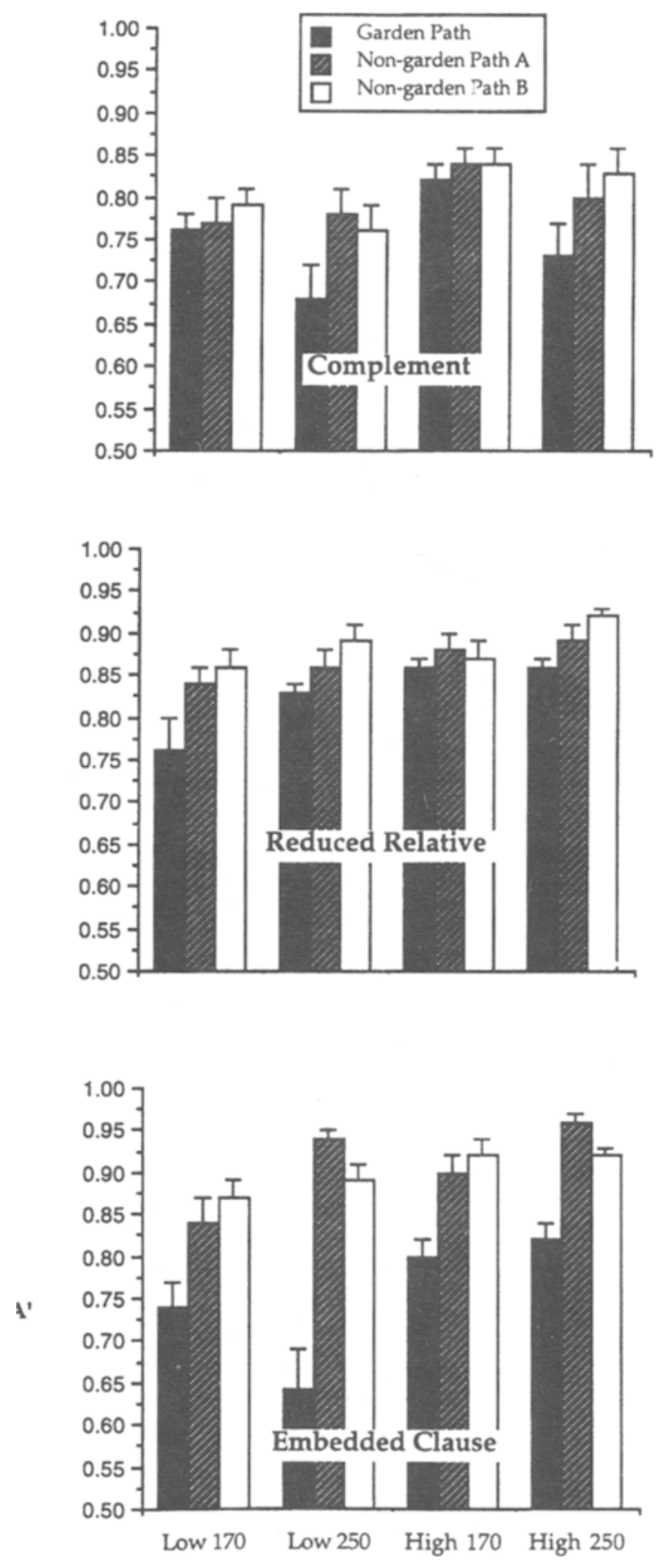

Figure 5. Mean $A^{\prime}$ scores (and standard deviations) for each memory span group (low, high) and sentence type at each presentation rate in Experiment $2 \mathrm{~A}$.

action was due to a somewhat different pattern of performance across the three structures for garden path, non-garden path A, and non-garden path B sentences. ${ }^{4}$ In addition, there was a significant interaction between memory span and garden path status $\left[F_{1}(2,90)=5.7, p<.01 ; F(2,252)=25.6\right.$, $p<.001]$. Analysis of this interaction showed that both memory span groups showed the garden path effect outlined above (garden path $>$ non-garden path $\mathrm{A}=$ non-garden path $B$ ). The interaction was due to the reaction times of the 

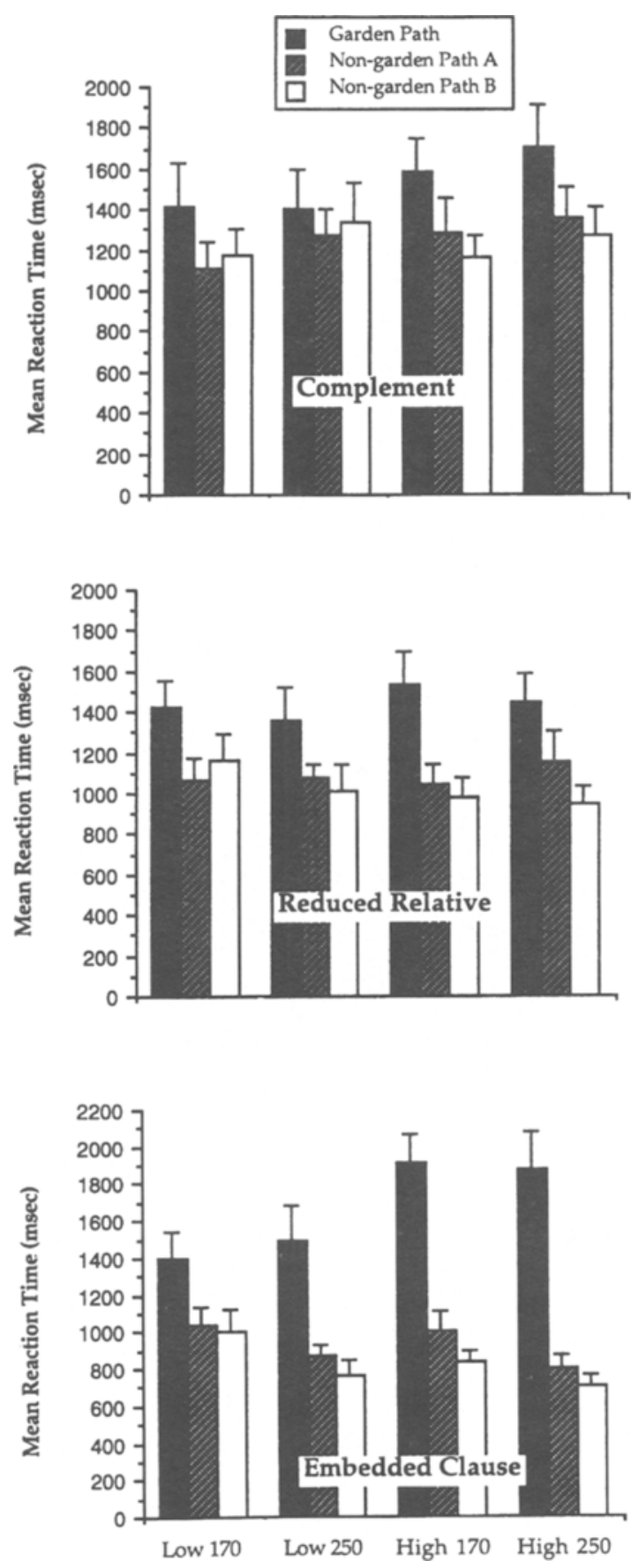

Figure 6. Mean reaction times (and standard deviations) for correct responses for each memory span group (low, high) and sentence type at each presentation rate in Experiment $2 \mathrm{~A}$.

high-span subjects' being longer than those of the low-span subjects on garden path sentences but not on either of the two types of non-garden path sentences.

\section{Experiment 2B}

Discrimination of acceptable and unacceptable sentences. Mean $A^{\prime}$ scores are shown in Figure 7. A 2 (memory span) $\times 3$ (structure) $\times 3$ (garden path status) ANOVA was carried out on the $A^{\prime}$ scores. Low-span subjects (mean $A^{\prime}=.71$ ) were less sensitive to the acceptability of a sentence than were high-span subjects $(.81)[F(1,30)=9.8$, $p<.01]$. Subjects were less sensitive to the acceptability of sentences with complement structures (mean $A^{\prime}=.72$ ) than to either of the other two structures, which did not differ from one another (.78 for both) $[F(2,60)=32.9, p<$ $.001]$. In addition, subjects were less sensitive to the acceptability of garden path sentences (mean $A^{\prime}=.72$ ) than to either type of non-garden path sentence, and $A^{\prime}$ scores for non-garden path A sentences (.76) were significantly lower than for non-garden path B sentences $(.79)[F(2,60)$ $=19.8, p<.001]$. The main effect of garden path status was qualified by an interaction with structure, which showed that only in the case of sentences with embedded structures were subjects less sensitive to garden path sentences than to the non -garden path sentences $[F(4,120)=$ $4.7, p<.001]$.

Reaction times for acceptable sentences. Mean reaction times for correct responses are shown in Figure 8. Reaction times were significantly longer to garden path than to either type of non-garden path sentence and the two types of non-garden path sentences did not differ $\left[F_{1}(2,60)=\right.$ $\left.25.7, p<.001 ; F_{2}(2,261)=30.9, p<.001\right]$. However, a significant structure $\times$ garden path status interaction showed that this pattern held only for sentences with reduced relative and embedded structures $\left[F_{1}(4,120)=6.5, p<.001\right.$; $\left.F_{2}(4,261)=4.8, p<.001\right]$. There were no significant garden path effects for sentences with complements. In addition, a significant memory span group $\times$ garden path status interaction showed that the high-span subjects showed garden path effects in relation to both non-garden path $\mathrm{A}$ and non-garden path $\mathrm{B}$ sentences $\left[F_{1}(2,60)=3.8, p<.05\right.$; $\left.F_{2}(2,261)=14.3, p<.001\right]$, while the low-span subjects showed garden path effects only when garden path sentences were compared with non-garden path B sentences.

\section{Discussion}

The purpose of these experiments was to determine whether subjects with low working memory capacities would be differentially impaired under conditions that have been claimed to increase processing load. In particular, we were interested in whether differences between high- and low-span subjects would begin to emerge with faster RSVP rates than that used in Experiment 1.

Comparison of the results from Experiment 1 and Experiments $2 \mathrm{~A}$ and $2 \mathrm{~B}$ shows that all of the basic phenomena found in Experiment 1 were replicated. All three studies showed that low-span subjects were less sensitive to the acceptability of a sentence than were high-span subjects, that sentences containing complement structures were harder than those with reduced relatives or embedded clauses, and that garden path structures were harder to process than non-garden path sentences. Thus, the results of Experiment 1 were replicated at faster RSVP rates.

Just and Carpenter's (1992) single-component capacity theory predicts that low-span subjects should have increased difficulty with sentence processing at faster presentation rates and particularly when the stimulus materials 
are more difficult. Contrary to the predictions of the capacity theory, the increased presentation rate in these experiments did not result in the low-span subjects' being more affected on the harder sentence types or on garden path sentences. Though there was a significant garden path status $\times$ memory span interaction in the reaction time data in Experiment 2B, there were garden path effects for both groups; the interaction resulted from differences in the groups with respect to whether the effect was signifi-
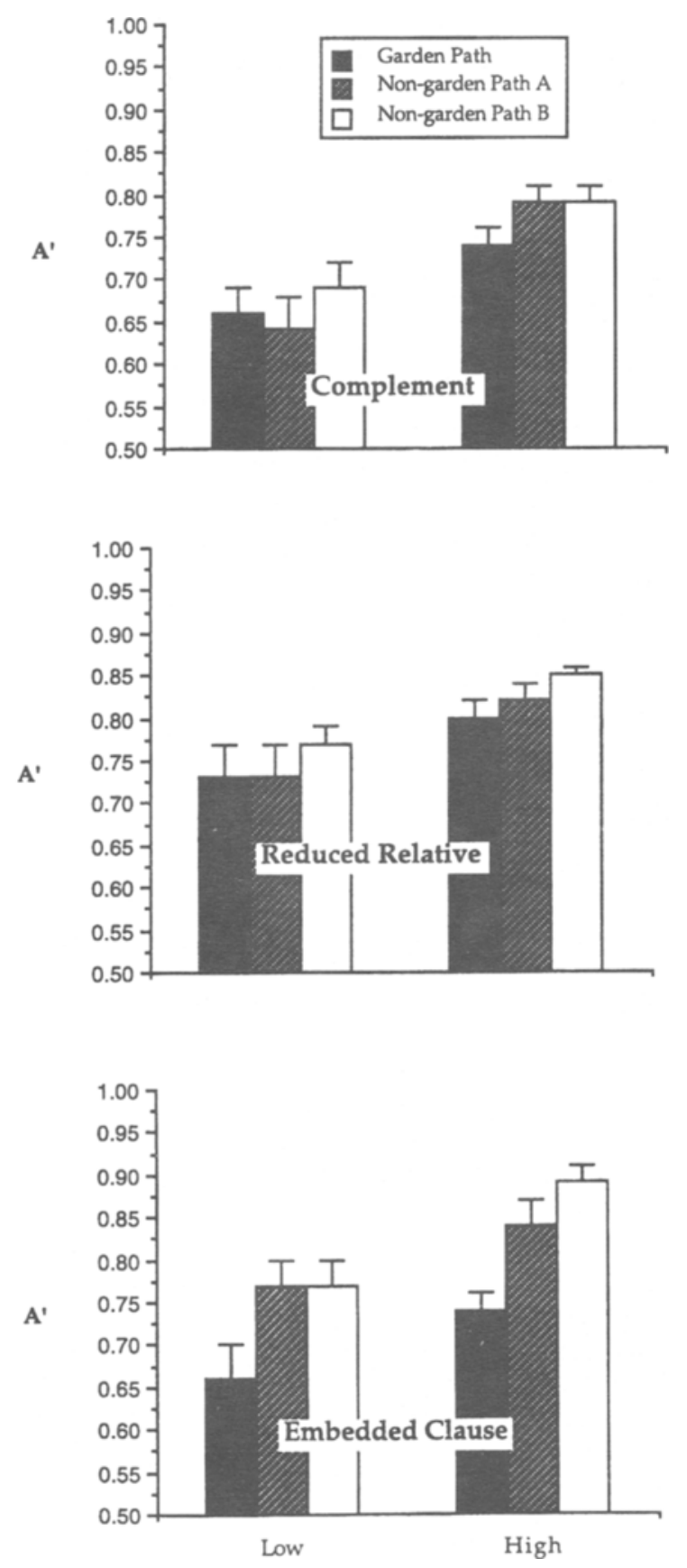

Figure 7. Mean $A^{\prime}$ scores (and standard deviations) for each memory span group (low, high) and sentence type in Experiment 2B.
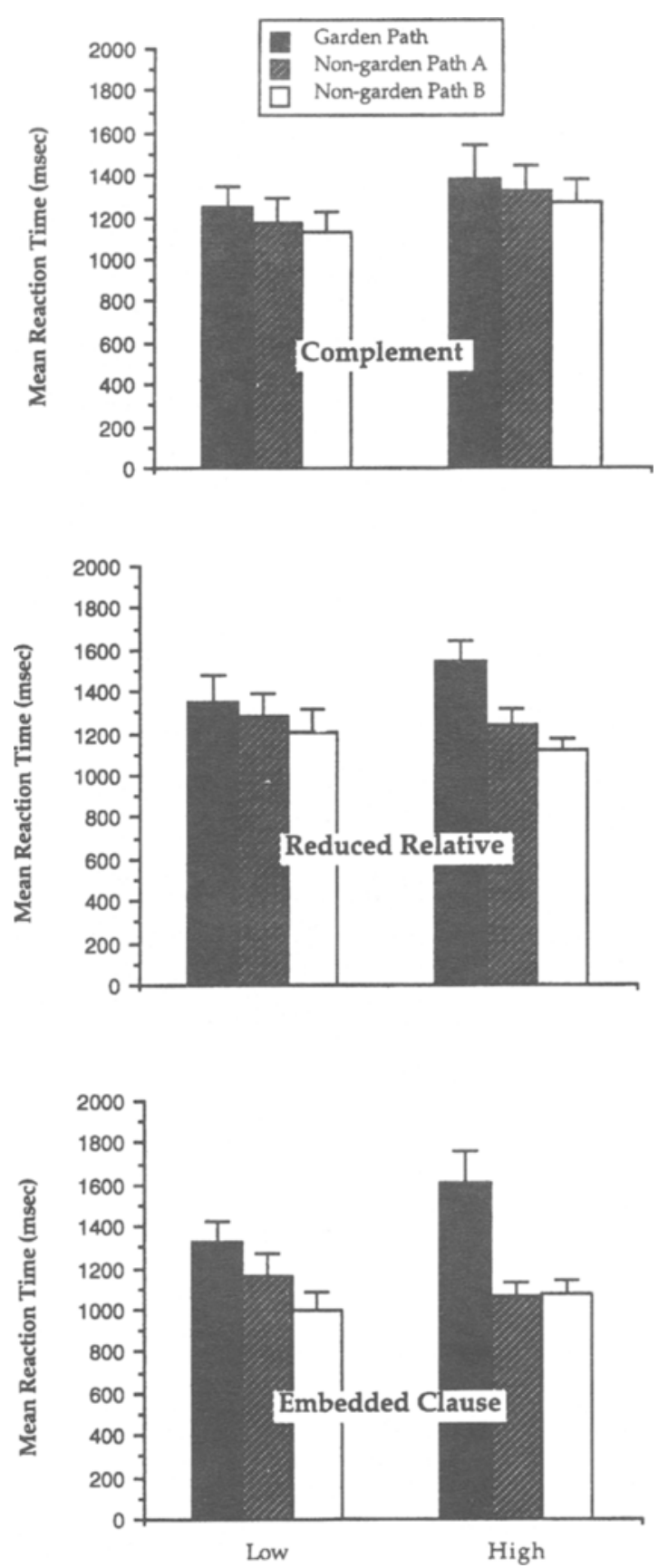

Figure 8. Mean reaction times (and standard deviations) for correct responses for each memory span group (low, high) and sentence type in Experiment $2 B$.

cant when garden path sentences were compared with both types of non-garden path sentences or only with one type. The low-span subjects were not significantly more affected by garden path structures at the fast RSVP rate.

One might still question whether the presentation rates used in these experiments were sufficiently taxing to bring out group differences. In fact, the evidence that the faster RSVP rates were more demanding than the slower rates is equivocal. The mean $A^{\prime}$ score in the $120-\mathrm{msec}$ presenta- 
tion condition in Experiment 2B (.76) was lower than those in the 250 -msec presentation conditions of Experiment 1 $(.89)$ or $2 \mathrm{~A}(.83)$, and lower than that in the 170 -msec presentation condition of Experiment $2 \mathrm{~A}(.83)$. This finding supports the claim that the faster presentation rate made the task more difficult. However, in Experiment 2A, there was no significant main effect of presentation rate on either $A^{\prime}$ 's or reaction times. $A^{\prime}$ scores were higher for the more difficult complement structure at the 250 -msec presentation rate than at the $170-\mathrm{msec}$ rate, but there were longer reaction times for the more difficult complement structure than for the other structures in the slower $250-\mathrm{msec}$ presentation condition and not in the faster $170-\mathrm{msec}$ presentation condition. In addition, garden path effects were seen for both complement and embedded structures at the slower 250-msec presentation rate, but only for embedded structures at the faster $170-\mathrm{msec}$ rate. These results raise the question of whether the faster RSVP rate was more demanding than the slower one.

In considering this question, we must bear in mind that different RSVP rates would be expected to affect processing in complex ways. Compared with whole-sentence written presentation, RSVP increases certain aspects of the memory load associated with sentence processing, because it requires that a subject retain the words of a sentence in memory. However, a faster RSVP rate can have mixed effects compared to a slower one. On the one hand, it can increase processing load if words are not recognized, because a subject must attempt to infer a missed word or access it from a prelexical representation that is held in memory. On the other hand, if words are recognized, a faster RSVP rate can reduce the total time during which they must be held in memory. Finally, a very fast RSVP rate could exceed the rate at which lexical information can be used to structure a sentence. These different effects probably led to the different effects of faster RSVP rates on more complex sentence types and garden path sentences in specific experiments. The fastest RSVP rate used here is comparable to the fast RSVP rate used in other research dealing with individual differences in working memory on sentence processing (Miyake et al., 1994). Though the effects of different RSVP rates are not fully understood, the use of a variety of rates serves to increase processing load in comparison with the usual whole-sentence reading task in different ways. Thus, the failure to find a reliable difference between high- and low-span subjects' abilities to make acceptability judgments about garden path sentences at any of the RSVP rates used here supports the view that low-and high-span subjects do not differ in their abilities to process garden path sentences under more demanding task conditions.

\section{GENERAL DISCUSSION}

The purpose of these experiments was to test predictions made by Just and Carpenter's (1992) capacity theory of language comprehension. As outlined in the introduction, according to this theory, individual differences in a single-component verbal working memory capacity are a major determinant of subjects' ability to determine sentence structure. Thus, individuals with lower working memory spans are claimed to be less efficient at carrying out a variety of syntactic operations, particularly when the stimulus materials or the task are more demanding.

One piece of data that has been argued to provide support for the capacity theory is the alleged finding that lowspan subjects have more difficulty than high-span subjects have in processing garden path structures (MacDonald et al., 1992). Given the many limitations of the empirical data that have been provided to support this claim, one purpose of the present study was simply to determine whether subjects who differed in working memory capacity would show robust and reliable differences in their abilities to process garden path structures. In addition, we were interested in whether these differences between groups would be seen with the use of a variety of different syntactic structures that have been shown to result in garden path effects. Finally, we were interested in whether, if such differences did exist, they would be exacerbated under experimental conditions that placed greater demands on processing resources.

In the studies presented here, 177 subjects who differed in working memory capacity were tested on their ability to make acceptability judgments about 450 sentences that differed in terms of their syntactic structure, whether or not they contained a garden path structure, and whether or not they were acceptable. None of the experiments found support for MacDonald et al.'s (1992) claim that high- and low-span subjects differ in their ability to process garden path structures. This was true regardless of whether subjects were tested on sentences with reduced relative structures, as in the MacDonald et al. study, or on longer and more difficult structures-complements and embedded clauses. Although all of the structures used in these experiments produced reliable and robust garden path effects, in no case were these effects any greater in subjects with lower working memory spans. This basic finding held regardless of whether the subjects were college students, as in the MacDonald et al. study, or normal elderly subjects, and regardless of the exact nature of the task used to divide subjects into the different memory span groups. It is important to keep in mind that, while these results depart from MacDonald et al.'s claims, they do not actually differ from their data, since, as noted in the introduction, MacDonald et al. failed to find statistically significant differences among the subject groups in their study. Together, these results suggest that low-span subjects are not less efficient than high-span subjects at processing garden path sentences.

One possible explanation for the failure to find the predicted interactions between working memory capacity and the ability to process garden path structures is that the experimental technique we used may have missed the effect of working memory capacity on sentence processing because it relied on an end-of-sentence measure of sentence processing. MacDonald et al. (1992) have suggested that the effect of differences in working memory capacity may only be seen during a short period of sentence processing, which may have been over at the point when subjects re- 
sponded to the acceptability of a sentence. However, garden path effects were most evident in the MacDonald et al. study on the measure taken at the end of the sentence. Furthermore, in this study, garden path effects were very strong at the point that subjects responded in the acceptability judgment task. This provides reason to believe that the processing load imposed by the garden path structures was still present at the point where subjects responded. If this load could be better borne by one group of subjects, that group should show less effect of garden path structures, but it did not.

The second major finding was that the RSVP presentation conditions failed to differentially exacerbate the effects of garden path structures in low-span subjects. This was true despite the evidence that the RSVP presentation condition made the sentence acceptability judgment task more difficult overall: $A^{\prime}$ scores were lower in the RSVP condition than in the whole-sentence condition and were poorer across experiments as the presentation rate increased. The failure to find differences in the efficiency of high- and low-span subjects in processing garden path sentences held across three RSVP rates. Even though faster RSVP rates affect subjects in complex ways, this finding indicates that subjects with lower working memory spans are able to process these sentences under a variety of demanding task conditions.

The effect of different RSVP rates on high- and lowspan subjects' abilities to process syntactic structures has been investigated for other sentence types by Miyake and his colleagues (Miyake, 1994; Miyake et al., 1994). These researchers presented sentences that differed in syntactic form to subjects with high, medium, and low reading spans under different RSVP rates and tested subjects' knowledge of the thematic roles in each sentence. Miyake et al. found significant interactions between span group and sentence type and between span group and RSVP rate. The span group $\times$ RSVP rate interaction shows that subjects with lower working memory spans were less capable of handling the RSVP condition. The span group $\times$ sentence type interaction shows that subjects with different working memory spans performed differently on different sentence types. However, in a reanalysis of data from Miyake (1994, Experiment 3 ), we have found that there was no significant difference between low- and high-span subjects' performances on the syntactically more complex sentences (Caplan \& Waters, 1995). Miyake and his colleagues also did not find a three-way interaction between span group, RSVP rate, and sentence type. Thus, the results of the studies by Miyake and his colleagues are consistent with ours in suggesting that high- and low-span subjects are equally capable of processing syntactically more complex sentences, even under demanding task conditions.

A final issue is the nature of the processing that is involved in comprehending garden path sentences. A "modular" view of sentence processing maintains that garden path sentences are more difficult because only the preferred structure is initially constructed and the sentence must be reviewed and its syntactic structure reassigned when the reader experiences the garden path effect (Frazier, 1990).
If this view is correct, these experiments suggest that lowspan subjects can accomplish this review and revision as well as high-span subjects. A second model maintains that garden path sentences are more difficult because two structures - the preferred as well as the unpreferred structure-are initially constructed (MacDonald et al., 1992). If the additional complexity is due to subjects' maintaining two structures, low-span subjects appear to be as capable of doing this as high-span subjects. A third view maintains that the assignment of thematic roles is accomplished by a process that satisfies constraints imposed by the morphology and semantics of the words in a sentence, and that this lexical information is less constraining in garden path than in non-garden path sentences (MacDonald, 1994; MacDonald, Pearlmutter, \& Seidenberg, 1995). If this is how sentences are processed, this constraint satisfaction process seems to proceed as well in low- as in high-span subjects, at least for garden path sentences.

In summary, the results of these experiments suggest that low-span subjects can process garden path sentences as well as high-span subjects can. One must, of course, interpret null results with caution. However, in the present experiments we tested a large number of subjects in each span group, and we used many sentences of each type. The expected results of garden path structure and of RSVP condition were found, indicating that the methods were sensitive to the independent variables that usually affect them. The results are consistent across whole-sentence presentation and RSVP presentation at three rates, in three different groups of subjects that differ in age. They are also consistent with the findings reported by MacDonald et al. (1992) regarding performance of subjects with different spans on garden path sentences, and with a reanalysis of results reported by Miyake (1994) on the effects of syntactic structure on sentence processing in subjects with different spans under fast RSVP conditions.

If we therefore (cautiously) accept the failure to find different effects of garden path status in low- and high-span groups as a basis for theory construction, these experiments speak against Just and Carpenter's (1992) capacity theory. The capacity theory asserts that (1) assigning more complex syntactic structures, comprehending sentences under RSVP conditions, and achieving a higher score on the Daneman and Carpenter (1980) working memory task all require more processing resources, and (2) these resources all come from the same pool. If these premises are correct, subjects with low Daneman and Carpenter working memory spans should perform less well than highspan subjects on garden path sentences, and this effect should be greater under RSVP than under whole-sentence presentation. These predictions are not supported by our results. Though there were interactions involving the group factor and garden path status, analyses of the terms of these interactions show that in none of them were low-span subjects significantly less able than high-span subjects to process garden path sentences.

We are therefore led to the conclusion that one of the premises of the capacity theory is incorrect. We suggest that what should be abandoned is the claim that the re- 
sources measured by the Daneman and Carpenter (1980) reading span tasks and those used in assigning the syntactic structure of a sentence all come from the same pool. We suggest that the working memory capacity that is measured by the Daneman and Carpenter span task is relevant to a class of verbally mediated tasks, but not to unconscious, obligatory language processing operations such as recognizing and understanding words and assigning the structure and the literal meaning of sentences. The last of these functions appears to utilize a specialized set of processing resources. The cognitive functions that make use of the processing resources that are measured by the Daneman and Carpenter span task remain to be precisely characterized. It is possible that they include verbally mediated reasoning and problem solving, or that they are ones that require conscious, controlled processing. Further research will be required in order to understand the structure of the verbal processing resource system and how different components of this system are used in different tasks.

\section{REFERENCES}

Altmann, G., \& Steedman, M. J. (1988). Interaction with context in human syntactic processing. Cognition, 30, 191-238.

BEVER, T. G. (1970). The cognitive basis for linguistic structures. In J. R Hayes (Ed.), Cognition and the development of language (pp. 279-362). New York: Wiley.

Caplan, D., Baker, C., \& Dehaut, F. (1985). Syntactic determinants of sentence comprehension in aphasia. Cognition, 21, 117-175.

Caplan, D., \& Hildebrandt, N. (1988). Disorders of syntactic comprehension. Cambridge, MA: MIT Press, Bradford Books.

CAPLAN, D., \& WATERS, G. S. (1994, October). Syntactic processing in sentence comprehension by aphasic patients under dual-task conditions. Paper presented at the Academy of Aphasia, Boston.

Caplan, D., \& Waters, G. S. (1995). Aphasic disorders of syntactic comprehension and working memory capacity. Cognitive Neuropsychology, 12, 637-649.

Daneman, M. (1994, July). Working memory and language: $A$ metaanalysis. Paper presented at the International Conference on Working Memory, Cambridge, England.

DANEMAN, M., \& CARPENTER, P. A. (1980). Individual differences in working memory and reading. Journal of Verbal Learning \& Verbal Behavior, 19, 450-466.

FODOR, J. (1983). The modularity of mind. Cambridge, MA: MIT Press, Bradford Books.

FraziER, L. (1990). Exploring the architecture of the language processing system. In G. T. M. Altmann (Ed.), Cognitive models of speech processing: Psycholinguistic and computational perspectives (pp. 409433). Cambridge, MA: MIT Press, Bradford Books.

Just, M., \& CARPENTER, P. A. (1992). A capacity theory of comprehension. Psychological Review, 99, 122-149.

KING, J., \& JUST, M. A. (1991). Individual differences in syntactic processing: The role of working memory. Journal of Memory \& Language, 30, 580-602.

MacDonald, M. C. (1994). Probabalistic constraints and syntactic ambiguity resolution. Language \& Cognitive Processes, 9, 157-201.

MacDonald, M. C., Just, M., \& Carpenter, P. A. (1992). Working memory constraints on the processing of syntáctic ambiguity. Cognitive Psychology, 24, 56-98.
Macdonald, M. C., Pearlmutter, N., \& Seidenberg, M. S. (1995). The lexical nature of syntactic ambiguity resolution. Unpublished manuscript.

MIYAKE, A. (1994). A resource-reduction theory of syntactic comprehension disorders: Making normal adults perform like aphasic patients. Unpublished doctoral dissertation, Carnegie Mellon University.

Miyake, A., Carpenter, P. A., \& Just, M. A. (1994). A capacity approach to syntactic comprehension disorders: Making normal adults perform like aphasic patients. Cognitive Neuropsychology, 11, 671-717.

POLLACK, I., \& NORMAN, D. A. (1964). A non-parametric analysis of recognition experiments. Psychonomic Science, 1, 125-126.

Rochon, E., Waters, G. S., \& Caplan, D. (1994). Sentence comprehension in patients with Alzheimer's disease. Brain \& Language, 46 , 329-349.

Trueswell, J. C., Tanenhaus, M. K., \& Garnsey, S. M. (1994). Semantic influences on parsing: Use of thematic role information in syntactic ambiguity resolution. Journal of Memory \& Language, 33, 285-318.

Wanner, E., \& Maratsos, M. (1978). An ATN approach to comprehension. In M. Halle, J. Bresnan, \& G. A. Miller (Eds.), Linguistic theory and psychological reality (pp. 119-161). Cambridge, MA: MIT Press.

WATERS, G. S., \& CAPLAN, D. (in press). The capacity theory of sentence comprehension: A reply to Just \& Carpenter. Psychological Review.

Waters, G. S., Caplan, D., \& Hildebrandt, N. (1987). Working memory and written sentence comprehension. In M. Coltheart (Ed.) $A t$ tention and performance XII: The psychology of reading (pp. 531555). Hove, U.K.: Erlbaum.

Waters, G. S., CaPlan, D., \& Rochon, E. (1995). Processing capacity and sentence comprehension in patients with Alzheimer's disease. Cognitive Neuropsychology, 12, 1-30.

\section{NOTES}

1. This task is similar to that used in Daneman and Carpenter (1980), Experiment 2, in which subjects made true-false judgments for sentences and recalled their final words. Daneman and Carpenter found correlations of .80 to .88 between their original oral reading span task and tasks such as this one in which subjects were required to make judgments about the sentences rather than simply read them aloud.

2. Analysis of these scores did not include data for the 9 subjects whose working memory spans were 1.0 or 1.5 , since these subjects were only tested at Span Size 2. In addition, owing to a computer malfunction, comprehension scores were unavailable for 5 subjects at span.

3 . The data were also analyzed when the subjects were divided into three groups on the basis of age $(50-59,60-69,70-80)$. The three groups did not differ in terms of years of education or WAIS vocabulary scores. However, somewhat unexpectedly, they also did not differ in terms of working memory span. Moreover, analysis of the data from the experimental tasks showed that there were no significant main effects of age or interactions with age in any of the analyses.

4. The interaction was due to the following pattern: (1) the difference between complements and reduced relatives was not significant for either garden path or non-garden path A structures, but was significant for non-garden path B structures; (2) the difference between reduced relatives and embedded structures was significant for garden path and nongarden path B structures but not for non-garden path A structures; and (3) the difference between complement and embedded structures was not significant for garden path sentences but was significant for both types of non-garden path structures.

(Manuscript received January 18, 1995; revision accepted for publication May 16, 1995.) 